\title{
Analysis of Training Methods of Table Tennis Players' Linking Technology
}

\author{
Xiaoyu Tian \\ Changchun Institute of Technology, Changchun, Jilin, China, 130600
}

Keywords: Training Methods, Table Tennis, Players' Linking Technology

\begin{abstract}
Table tennis convergence technology is to train athletes to master the "line changes" laws and active defense tactics to predict each other's return line, and move ahead to the best batting hit the ball to achieve. Interactive multi - point and multi - ball training is an effective way to cultivate amateur line change consciousness.
\end{abstract}

\section{Introduction}

Amateur athletes often complain, why I played for many years of table tennis, but the ball hit a certain degree of technical level after the stagnation of it? In contrast, professional athletes match the ability of the game is very strong, as if to predict the other side of the ball. Why can they play so many rounds? In fact, the existence of this idea amateur athletes are common, they are mainly lack of professional athletes "convergence" consciousness.

Professional athletes on the second board forehand short ball back to the other side of the hand position (or the other backhand) near the network has been set in advance of its backhand with the gap of this trap, lure the other back to its backhand position, and then 4 board quickly sideways hair force straight line (or sideways pull slash), and then cross the flop right side of the first board 6 (or red line) and so on. This is "the athlete designed in advance routines, intends to attack each other the opportunity to predict each other's back to the ball line, move ahead of time to the best batting hit the ball in the defense to win or for the attack to create greater possibilities". This play called active defense tactics. From the convergence of this sense, it and the "line change" law has the same purpose. Professional athletes rely on this "line changes" and "active defense" awareness to predict the other side of the next board of the ball back line. Another example, when playing the ball, the other in the case of unprepared, if the forehand catch, back to the middle or the middle of the road is more common, back to the big hand of the possibility of less (with backhand When similar).

"Line changes" and "active defense" of the war there are many, these are more typical, in actual combat can often see and there is a high probability of universal technical and tactical. So it can be said that this is the law. Professional athletes are using this law to master the convergence of technology, in order to achieve a number of rounds of the contest.

\section{The Table Tennis Player Convergence Technology}

The so-called "convergence" is the athletes in accordance with the table tennis "line changes" rule to predict each other's return line, and move ahead of time to the best batting hit the ball. Such as professional athletes to play backhand, B player second board backhand pick up the ball grab slash and A athletes on the 3rd board backhand to form a slash; when B 4 board becomes straight, A will be the first 5 board habitual forehand Slash (this probability is very high). At this time B board 6 board has been waiting for a straight line in the forehand. This is called backhand straightened straight hand. B 4 and 6 board why can be so tacit understanding? The reason is very simple, B this line of change is the use of A play the habit of thinking fixed potential (every straight variable slope) to achieve.

\section{The Table Tennis Players "Line Changes" Rule}

"Line change" rule is the people of the table tennis five basic lines (forehand bit oblique, straight; 
backhand bit oblique, straight; middle bit line) repeated practice, from practice to explore out. First of all, people use the same direction of technology to play called "with the line back" technology, and then explore the ability to mobilize each other to create a favorable situation for their own "close-line" technology called "every oblique straight" and " "technology. It can be said that this is the most primitive tactics of table tennis. Through it can evolve a variety of tactics. Such as "right shift left", "hit the middle to kill two corners", "playing two points to kill the road" and so on. People in the long-term use of "every oblique straight" and "every straight" tactical process found that it is very practical and regular to follow, so it can be cured into a way of thinking (forming a sense). It can be said that this is one of the "line changes" rule. No matter what kind of tactics used by athletes, are around the "every oblique straight" and "every straight ramp" two basic technical tactics to play line changes. Therefore, the professional athletes on this basis, the "forehand slanting straight into the back of the straight line to play, backhand slash phase change straight forward hit", "forehand or backhand when holding the middle and then hit the two horns" Backhand slash holding side body attack and then flutter right "as the law of the line to use. In fact, this is the use of the other side of the mindset at the same time, he also put it into a regular, fixed play mode, is the embodiment of the concept of integration. Master it will form a "line change" consciousness. In order to facilitate future training "line change" consciousness, the author of these three kinds of technical tactics of the first two known as "close to Taiwan" technical and tactical.

From the table tennis tactical point of view, "close to Taiwan" is the tactics, the use of more. And "active defense" is the dark tactics, although it has the effect of convergence before and after the two plates. But to take a certain risk cannot be used. Athletes in the use of convergence technology can be considered preferred the former, the latter election. The combination of the two is a wise move, the formation of complementary advantages.

It should be noted that the "line change" this law is not static, we can use the "same line back" technology to break it. As in the case of the above-mentioned $\mathrm{B}$ athletes backhand slash to straighten the straight line to keep the hand with more, A athletes will consciously change their mindset and use the "same line back" so that B athletes in the forehand position, It can produce anti-conventional tactical effect.

\section{The Table Tennis Athletes Convergence Technical Training Methods}

Interactive Multi-Point Multi-Ball Training Method. The traditional multi-point multi-ball training method is the main coach, designed to send content to the ball for the players to play with the ball. And interactive multi-point multi-ball is the coach in accordance with the rules of the table tennis game line design changes to the main athletes: when the athletes change the line, the coach in accordance with the rules of the game with the change (for the ball), or coach for the ball changes when the line athletes Follow the rules of the game with the change (play). This training method is the coach with more balls and athletes competition, more than for the point of the multi-point training more accurate and effective, so that athletes have their own ball after the feedback to the ball, coaches between the ball and the ball there is an internal link, the ball is not a small change, very suitable for training athletes line change awareness training.

Training analysis: amateur athletes generally backhand holding the sideways forehand use very little or only sideways to play, but not commonly used, the game even more afraid to use. The reason is that they are not subject to orthodox footwork training, sideways forehand to hit the forehand position of the risk of the problem, if not cross-flop right will directly affect the quality of sideways to fight (confused). So amateur athletes must learn to cross the right flop in order to have sideways to fight the emboldened in order to appreciate the backhand to hold more sideways to use the benefits of forehand play, which is an effective way to improve the ability to support backhand. Because the power of the forehand is much larger than the backhand, can play a strong rhythm changes and make the other mistakes. Backhand hand-held sideways forehand and fight with the right to fight the problem of convergence, I believe that the backhand holding the sideways forehand does not have to flutter right, if the sideways to find the force of the ball, the quality of the ball must be high. The other is not wrong, then the same line with the probability of more than a 
straight line, so do not have to fight right. Similarly, if the sideways cannot find the force hit the ball, then the quality of the ball must not be high. The probability of a straight line to the other side is very high, it must be flop right to play, which is predicting the backhand hand-held sideways forehand after the cross-flop right-hand cohesion method.

Training points: cross-flop right for amateur athletes is a problem, first of all to know cross-flop right to play two techniques, " $4+2$ sideways flapping right" and "2 +4 sideways flapping right" difference. And then through the interactive multi-point multi-ball training, this problem can be solved. The former cross-flop right flush is greeting the front of the ball, left foot at the same time, the right hand to be synchronized in the ball after the rise of the late (2), and even hand faster than the foot, there is a moment of volley, and then the right Feet only landing. The right hand and left foot cannot be synchronized, but the right hand to pull the ball than the right foot floor to early or synchronous, technical requirements lower than the former. Commonly used in the transition when the offensive and defensive transition board (sideways grab a straight line, but the other side back to the slant space).

"Multi-Ball Single Practice" Training Method. In the above interactive multi-point multi-ball training on the basis of the re-applied "multi-ball single practice" means can achieve a multiplier effect. Because the practice of multi-ball single practice very strong confrontation, and can be combined with actual combat, the difficulty and strength of training sometimes even more than the game, is to improve the technical level of the athletes rare training means.

The so-called multi-ball single practice is to take one to one, one party to defend the team, the third party for the two sides in accordance with the technical or tactical requirements, starting from the actual, repeated practice, the players do not need to check the ball, Followed by the next ball practice. To improve the anti-pull the other side of the pull from the next pull up the rotation of the ball and the ball in the back of the ball with the ability to exercise the prescription (COSCO).

Training methods: multi-ball single practice ( $\mathrm{C}$ for short under the fixed penalty to A full Taiwan, A full hand split the other side of the B-hand position, so that both sides of the formation of the hand $\rightarrow$ pull +3 , there is no primary and secondary points, A, B, C must be rotated for a multi-ball; the other two to play the ball, until the ball and then the next round of practice. This training content is essentially the practice of the arc circle.

Example Analysis: This is a comprehensive ability of training, through this training can master the split long, pull the Canadian, pull the other side from the next pull up the rotation of the circle and improve the forehand near, in the far stage support ability. The focus of this training in the A athletes pull each other from the next pull up the turn of the circle of the ball, practice the technology (anti-pull the second point that is the rise), behind the continuous pull capacity will be solved. Athletes do not be intimidated by the content of this exercise, the beginning of the practice may soon be wrong, but after a period of practice will be able to pull more. This is a table tennis amateur master must master the convergence technology.

Multi-ball training is just table tennis teaching and training in the auxiliary practice methods, amateur athletes should also be based on single-ball practice. Multi-ball training and single-ball training time should be 1 : 2 . Usually after a multi-ball training, arrange for a certain period of time for regular single-ball training, for example, in the three training prescription behind the "single ball practice" to make up for the lack of training. All of the above training prescriptions can be changed, for example, in each training prescription slash "/" behind the contents of the design of the "variable form."

\section{Conclusion}

Table tennis convergence technology is to train athletes to master the "line changes" laws and active defensive tactics to predict each other's return line, and move ahead to the best hit the ball to achieve. Interactive multi - point and multi - ball training is an effective way to cultivate amateur line change consciousness. This paper mainly introduces the interactive multi-point multi-ball training method and the "multi-ball Single practice" training method, hoping to provide a certain 
reference for the improvement of the convergence technology of table tennis players.

\section{References}

[1] Zhong Yujing, Wang Dazhong in the table tennis in the "active defense"[J]. Table tennis world, 2008 (6): 21-22.

[2] Xiong Zhichao. Teaching design of multi-ball training in table tennis[J]. Journal of Physical Education, 2009, 16 (4): 76. 